\title{
Spectroscopic Identification of Isomeric Trimethylbenzyl Radicals Generated from 1,2,3,4-Tetramethylbenzene
}

\author{
Young Wook Yoon and Sang Kuk Lee* \\ Department of Chemistry and The Chemistry Institute for Functional Materials, Pusan National University, \\ Pusan 609-735, Korea. *E-mail: sklee@pusan.ac.kr \\ Received May 31, 2011, Accepted July 8, 2011
}

\begin{abstract}
The visible vibronic emission spectrum was recorded from the corona discharge of precursor 1,2,3,4tetramethylbenzene with a large amount of inert carrier gas helium using a pinhole-type glass nozzle coupled with corona excited supersonic expansion. The spectrum showed a series of vibronic bands in the $D_{1} \rightarrow D_{0}$ electronic transition of jet-cooled benzyl-type radicals formed from the precursor in a corona excitation. The analysis confirmed that two isomeric radicals, 2,3,4- and 2,3,6-trimethylbenzyl radicals, were produced as a result of removal of a hydrogen atom from the methyl group at different substitution positions. For each isomeric product, the electronic transition and a few vibrational mode frequencies were determined in the ground electronic state.
\end{abstract}

Key Words : Trimethylbenzyl radicals, Spectroscopy, Vibronic spectrum

\section{Introduction}

Whereas the benzyl radical, a prototypical aromatic radical, has been the subject of many spectroscopic studies, alkyl-substituted benzyl radicals have received far less attention, especially for polymethylbenzyl radicals due to the possible formation of several isomers from the precursor. ${ }^{1}$

Spectroscopic works on benzyl-type radicals in the visible region have been initiated by Schuler et $a .^{2}$ and Walker and Barrow. ${ }^{3}$ Bindley et al. ${ }^{4,5}$ have assigned several strong bands in the visible vibronic emission spectra of xylyl radicals produced from xylenes in a corona discharge. The technique of laser-induced fluorescence has been well applied by Charlton and Thrush $^{6}$ to determine the vibronic assignments and lifetime measurement of xylyl radicals.

Hiratsuka et $\mathrm{al}^{7}{ }^{7}$ have calculated the energies of closelying doublet states, $\mathrm{D}_{1}$ and $\mathrm{D}_{2}$ states in benzyl and xylyl radicals, and thereby explained the vibronic coupling between two electronic states. From the rotational contour analysis of the room temperature spectrum of the $o$-xylyl radical, Cossart-Magos et al. ${ }^{8}$ have determined the magnitude and direction of transition dipole moment change upon electronic excitation. High-resolution work has been carried out by Fukushima and $\mathrm{Obi}^{9}$ who have used the LIF technique to examine the vibronic coupling between two lowest excited electronic states of the jet-cooled p-xylyl radical. Bandshape analysis of high-resolution emission spectra satisfactorily resolved controversial vibronic assignments of $p$-xylyl radical. ${ }^{10}$ In addition, torsional analysis of the methyl rotor in xylyl radicals has been carried out by Lin and Miller. ${ }^{11}$ Selco and Carrick ${ }^{12,13}$ have assigned many bands in the vibronic emission spectra of benzyl and xylyl radicals.

For polymethylbenzyl radicals, Lee et $a l^{14-16}$ used an improved corona discharge system to observe the vibronic emission spectra of many species, from which each isomer generated from the precursor by removing a hydrogen atom from a different methyl group was clearly identified by analyzing the bandshape and the effect of methyl substitution on electronic transition.

The effect of methyl substitution on the electronic properties has been well investigated for polymethylbenzyl radicals by analyzing fluorescence spectra. In particular, by using first- and second-order perturbation theory, Petruska ${ }^{17}$ has derived general formulae showing that electronic transition of the parent hydrocarbon changes in intensity and shifts in frequency according to the nature, the number, and the position of the substituents on the ring. His results are generally consistent with the data obtained from the absorption spectra of these compounds.

The combination of corona discharge with supersonic expansion has proven to be a powerful spectroscopic tool for observing the vibronic emission spectra of transient molecular species. The spectral simplification and molecular stabilization associated with the expansion of a large amount of inert carrier gas cannot be achieved in any other way. Among the emission sources utilizing the combination, the pinhole-type glass nozzle provides sufficient continuous photon intensity for high-resolution studies of weak transition $^{18,19}$ in the gas phase and has been well adapted for observation of the vibronic emission spectra of benzyl-type radicals. $^{20}$

In the present study, we report the visible vibronic emission spectrum observed from the corona discharge of precursor 1,2,3,4-tetramethylbenzene, and assign the observed bands based on the substitution effect of xylyl radicals. From the analysis, we confirm the existence of two isomeric trimethylbenzyl radicals, 2,3,4- and 2,3,6-trimethylbenzyl in corona discharge, and determined the spectroscopic data in 
the $\mathrm{D}_{1} \rightarrow \mathrm{D}_{0}$ electronic transition as well as the vibrational modes in the ground electronic state.

\section{Experimental Section}

The experiment was carried out with the setup described in detail elsewhere. ${ }^{21}$ Briefly, a pinhole-type glass nozzle was employed to produce jet-cooled benzyl-type radicals in corona excited supersonic expansion (CESE) that coupled corona discharge with jet expansion. The vibronic emission spectra were recorded with a long-path monochromator in the visible region.

Electronically hot but rovibrationally cold benzyl-type radicals were produced from the corona discharge of precursor 1,2,3,4-tetramethylbenzene (Aldrich, reagent grade) seeded in a large amount of inert carrier gas helium. To improve the emission intensity, we increased the precursor concentration in the carrier gas by heating the sample to $50{ }^{\circ} \mathrm{C}$ and adjusted the mixing ratio by monitoring the emission intensity from the strongest band that was believed to be about $1 \%$ in the mixture. A $0.3 \mathrm{~mm}$ diameter, pinholetype glass nozzle was used to eject the gas mixture in corona discharge. A sharpened long stainless steel rod acting as an anode was connected to a high voltage dc power supply in a negative polarity, in which the axial discharging current was $5 \mathrm{~mA}$ at a $1.5 \mathrm{kV}$ dc potential, and stabilized using a $150 \mathrm{k} \Omega$ current-limiting ballast resister.

A six-way, cross-type expansion chamber made of thickwalled, $50 \mathrm{~mm}$ diameter Pyrex tubes was evacuated by using a $800 \mathrm{l} / \mathrm{min}$ mechanical rotary vacuum pump to give a pressure of 3.0 mbar during continuous expansion, with 3.0 bar of backing pressure that was limited by the pressure tolerance of the glass materials. A cathode in the form of a long copper rod was positioned parallel to the jet direction under the expansion chamber to avoid arc noise reaching the spectrometer.

A weak blue-green colored jet was evidence for the presence of the benzyl-type radicals of the $\mathrm{D}_{1} \rightarrow \mathrm{D}_{0}$ transition in the expansion. The light emanating from the downstream jet area $5 \mathrm{~mm}$ from the nozzle opening was collimated by a collecting quartz lens $(f=5.0 \mathrm{~cm}, \mathrm{D}=3.8 \mathrm{~cm})$ placed inside the chamber, focused to the slit of the monochromator (Jobin Yvon U1000) equipped with two 1800 lines/mm gratings, and detected with a cooled photomultiplier tube (Hamamatsu R649) and a photon counting system. During the scans, the slits were set to $100 \mu \mathrm{m}$, providing an effective resolution of about $2 \mathrm{~cm}^{-1}$ in the visible region. The spectral region from 18000 to $22000 \mathrm{~cm}^{-1}$ was singly scanned at $2.0 \mathrm{~cm}^{-1}$ increment over $2 \mathrm{hrs}$ to obtain the spectrum shown in Figure 1. The wavenumber of the spectrum was calibrated using the He atomic lines ${ }^{22}$ observed in the same spectral region as the benzyl-type radicals, and is believed to be accurate within $\pm 1.0 \mathrm{~cm}^{-1}$.

The $a b$ initio calculations were carried out on the ground electronic state $\left(\mathrm{D}_{0}\right)$ of the isomeric trimethylbenzyl radicals to assist the assignment of the vibronic structure of the spectrum. The calculations were performed with a personal

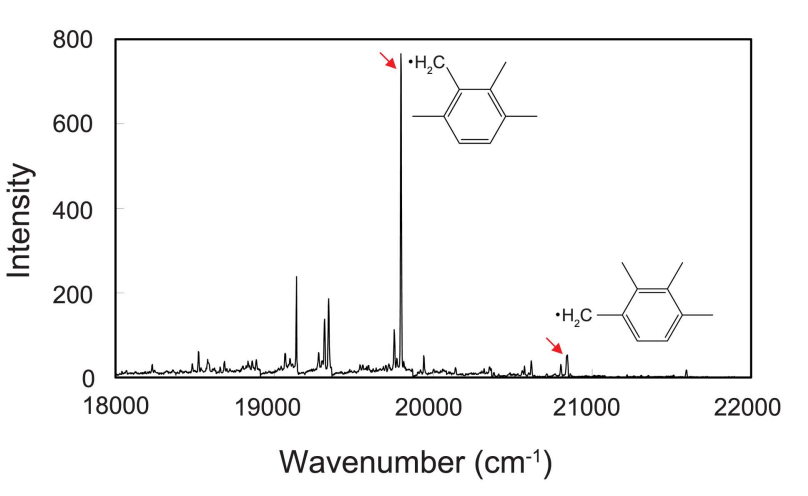

Figure 1. Visible vibronic emission spectrum observed from a corona discharge of precursor 1,2,3,4-tetramethylbenzene with a large amount of inert carrier gas helium in CESE. The assignment of each isomer is indicated by the molecular structure. The origin band of each isomer in the $\mathrm{D}_{1} \rightarrow \mathrm{D}_{0}$ electronic transition is marked by a red arrow.

computer equipped with an Intel Pentium IV CPU $2.80 \mathrm{GHz}$ processor with $2.0 \mathrm{~GB}$ RAM, according to the standard methods included in the Gaussian03 program. The geometry optimization and vibrational frequency calculations were performed at the DFT level, and the $6-311 G^{* *}$ basis set was employed in all of the calculations.

\section{Results and Discussion}

It has been demonstrated ${ }^{23}$ that a well-controlled corona discharge of substituted toluenes seeded in a large amount of carrier gas produces corresponding benzyl-type radicals in the excited vibronic state. Although the exact mechanism for the generation and excitation of benzyl-type radicals in corona discharge has not been established, it has been suggested that the metastable He atom ${ }^{24}$ in the $1 \mathrm{~s} 2 \mathrm{~s}^{3} \mathrm{~S}_{1}$ state, about $160,000 \mathrm{~cm}^{-1}$ above the ground state produced by corona excitation, transfers the excitation energy to the substituted toluenes through a collisional process, producing corresponding benzyl-type radicals by removing a hydrogen atom from the methyl group rather than the benzene ring. The mechanism for production of $o$-xylyl radical from $o$ xylene has been discussed in the liquid phase by laser excitation. $^{25}$ The excitation energy loosens one of the C-H bonds of the methyl group in the excited electronic state, eventually leading to the removal of a hydrogen atom from the methyl group.

Although the diradical ${ }^{26}$ has been reported to be produced in the matrix isolation from 1,3,5-trimethylbenzene, mesitylene, by extracting a hydrogen atom from each of the two methyl groups rather than from the benzene ring, diradicals in the gas phase have never been observed because of their extremely short lifetime and low concentration in the medium.

Since the molecule has a planar structure with 7 delocalized $\pi$ electrons, to which the $\mathrm{CH}_{2}$ group contributes a $\pi$ electron, the electronic interaction between the methyl group and the benzene ring is undoubtedly of the second order 
compared with that between the benzene ring and methylene group in methyl substituted benzyl-type radicals. Thus, the electronic structure of polymethylbenzyl radicals should exhibit a close relationship to that of the benzyl radical, and indeed one might be able to closely relate the two lowest lying excited electronic states of the polymethylbenzyl radicals to the parental benzyl radical $2^{2} \mathrm{~B}_{2}\left(\mathrm{D}_{2}\right)$ and $1^{2} \mathrm{~A}_{2}\left(\mathrm{D}_{1}\right)$ states. Thus, the weak visible emission transition of polymethylbenzyl radicals is believed to arise from the closelying $\mathrm{D}_{2}$ and $\mathrm{D}_{1}$ excited electronic states to the $1^{2} \mathrm{~B}_{2}\left(\mathrm{D}_{0}\right)$ ground state. Ring substitution would be expected to affect the energies of the two excited electronic states differently. The methyl group on the benzene ring could extend the delocalization space of $\pi$ electrons, making the energy of the excited electronic states be lower. The magnitude of energy lowering of the excited electronic states strongly depends on the number, the nature, and the position of the substituents on $\rightarrow$ the ring.

In benzyl-type radicals, two excited electronic states can be mixed through vibronic coupling that increases the relaxation rate of the population from $D_{2}$ to $D_{1}$ states. Therefore, the emission intensity of the $\mathrm{D}_{2} \rightarrow \mathrm{D}_{0}$ transition is inversely proportional to the relaxation rate that is related to the density of the vibrational states in the $\mathrm{D}_{1}$ state. The large separation of about $800 \mathrm{~cm}^{-1}$ between $\mathrm{D}_{2}$ and $\mathrm{D}_{1}$ states in the benzyl radical may include many vibrational levels, resulting in almost negligible emission intensity in the $\mathrm{D}_{2} \rightarrow$ $\mathrm{D}_{0}$ transition. The only exception in benzyl-type radicals is the strong observation of $p$-chlorobenzyl radical ${ }^{27}$ in the $\mathrm{D}_{2}$ $\rightarrow \mathrm{D}_{0}$ transition, which is attributed to the small difference of $95 \mathrm{~cm}^{-1}$ between the two excited electronic states.

Figure 1 shows a portion of the vibronic emission spectrum of the benzyl-type radicals formed by the corona discharge of precursor 1,2,3,4-tetramethylbenzene, prehnitene in which many of the strong bands are observable with a very good $\mathrm{S} / \mathrm{N}$ in the region of $19000-22000 \mathrm{~cm}^{-1}$. Whereas the benzyl radical exhibited the origin band at $22002 \mathrm{~cm}^{-1}$, the substitution of the methyl group(s) into the benzene ring showed red-shifted origin bands, as listed in Table 1. For mono-substituted benzyl radicals, the $o-, m-$, and $p$-xylyl radicals ${ }^{13}$ showed the origin bands at 21345 , 21485 , and $21700 \mathrm{~cm}^{-1}$, shifted by 657,517 , and $302 \mathrm{~cm}^{-1}$ from the benzyl radical, respectively, in the $D_{1} \rightarrow D_{0}$ electronic transition.

For polymethylbenzyl radicals, the origin bands were observed in the region as predicted by the substitution effect that had been used to identify the isomers generated from the precursor in a corona discharge. The large shift in dimethylbenzyl radicals with substitution at the ortho position is related to the $o$-xylyl radical that shows the largest shift among the isomers. The three isomeric dimethylbenzyl radicals ${ }^{28}$ generated in the corona discharge of 1,2,4-trimethylbenzene were assigned by substitution effect as well as bandshape analysis. The observation in the gas phase of the three isomers agreed well with that reported ${ }^{1}$ in the solid phase by within $500 \mathrm{~cm}^{-1}$, which was expected from the limited motion in the solid phase. In addition, the intensity
Table 1. Origin Bands in the $D_{1} \rightarrow D_{0}$ Transition of Methylsubstituted Benzyl Radicals ${ }^{a}$

\begin{tabular}{|c|c|c|}
\hline Molecules & Origin band $\left(\mathrm{cm}^{-1}\right)$ & Shift $\left(\mathrm{cm}^{-1}\right)^{h}$ \\
\hline benzyl $^{b}$ & 22002 & 0 \\
\hline$o$-methylbenzyl ${ }^{c}$ & 21346 & 656 \\
\hline$m$-methylbenzyl $l^{c}$ & 21486 & 516 \\
\hline$p$-methylbenzyl ${ }^{c}$ & 21700 & 302 \\
\hline 2,3-dimethylbenzyl ${ }^{d}$ & 20657 & 1345 \\
\hline 2,4-dimethylbenzyl ${ }^{d}$ & 20600 & 1402 \\
\hline 2,5-dimethylbenzyl ${ }^{d}$ & 20558 & 1444 \\
\hline 2,6-dimethylbenzyl ${ }^{d}$ & 20616 & 1386 \\
\hline 3,4-dimethylbenzyl ${ }^{d}$ & 21306 & 696 \\
\hline 3,5-dimethylbenzyl ${ }^{e}$ & 20842 & 1160 \\
\hline 2,4,5-trimethylbenzyl $f^{f}$ & 20832 & 1170 \\
\hline 2,3,4-trimethylbenzyl ${ }^{g}$ & 20844 & 1158 \\
\hline 2,3,6-trimethylbenzyl ${ }^{g}$ & 19796 & 2206 \\
\hline
\end{tabular}

${ }^{a}$ Measured in vacuum $\left(\mathrm{cm}^{-1}\right) .{ }^{b}$ Reference $12 .{ }^{c}$ Reference $13 .{ }^{d}$ Reference 28. ${ }^{e}$ Reference $15 .{ }^{f}$ Reference $14 .{ }^{g}$ This work. ${ }^{h}$ With respect to the origin band of benzyl radical $\left(22002 \mathrm{~cm}^{-1}\right)$.

and shape of the bands strongly depended on the symmetry of the species, reflecting the transition dipole moment of electronic and vibrational motion.

In the corona discharge of precursor 1,2,3,4-tetramethylbenzene, it is possible to generate two isomeric radicals, 2,3,4- and 2,3,6-trimethylbenzyl radicals, by eliminating a hydrogen atom from the methyl group at the 1- and 2position, respectively, as shown in Figure 2. Although the spectroscopic identification of large aromatic molecules of similar structure is not straightforward, the observation of the strongest origin band and a few vibronic bands of wellknown vibrational modes may provide more reliable evidence for the assignment of benzyl-type radicals. Nevertheless, the most trustworthy identification method is to analyze a highresolution spectrum exhibiting a rotational structure, as in the routine spectroscopic analysis of molecules. Since the spectrum obtained in this work showed a limited resolution and rotational temperature, we attempted to obtain the evidence of each isomer by comparing with those obtained from calculation and vibrational mode assignments.

From the solid state photolysis of 1,2,3,4-tetramethylbenzene, Branciard-Larcher and Migirdicyan ${ }^{1}$ have assigned

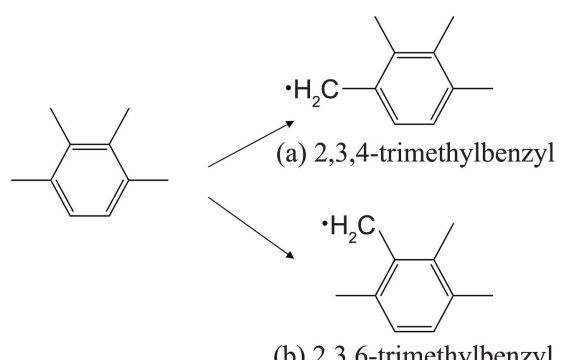

Figure 2. Two isomeric trimethylbenzyl radicals, (a) 2,3,4- and (b) 2,3,6-trimethylbenzyl radicals can be generated by removal of hydrogen atom from methyl group at the different position of precursor 1,2,3,4-tetramethylbenzene. 
the two broad bands observed in the visible region at 2.54 and $2.35 \mathrm{eV}$ to the origin bands in the $\mathrm{D}_{1} \rightarrow \mathrm{D}_{0}$ transition of 2,3,4-and 2,3,6-trimethylbenzyl radicals, respectively, by comparing with the result of a simple linear combination of atomic orbital (LCAO) self-consistent-field (SCF) calculation. However, the gas phase spectrum in Figure 1 exhibits three strong vibronic bands at 19342, 19796, and 20844 $\mathrm{cm}^{-1}$, which are possibly assigned as the origin band.

Since the emission spectrum observed in this work is similar to the single vibrational level-dispersed fluorescence spectrum obtained by exciting the origin band of the electronic transition, the spacing of the vibronic bands from the origin band represents the vibrational mode frequencies at the ground electronic state.

The vibronic emission spectra observed with a pinholetype nozzle in a CESE system always show the strongest origin band at the highest wavenumber for the benzyl-type radicals, because highly efficient vibrational relaxation in the $\mathrm{D}_{1}$ state increases the population in the vibrationless state. In addition, the similarity in molecular geometry between the two electronic states increases the intensity of origin band, owing to the large Franck-Condon integral. Thus, the vibronic band with the strongest intensity at 19796 $\mathrm{cm}^{-1}$ was assigned to the origin band of the 2,3,6-trimethylbenzyl radical because this radical showed a threefold greater oscillator strength than that of 2,3,4-trimethylbenzyl radical in the $D_{1} \rightarrow D_{0}$ electronic transition. ${ }^{1}$ Following this determination, the strong bands at 19342, 19316, and 19140 $\mathrm{cm}^{-1}$ were be assigned to the $6 \mathrm{a}_{1}^{0}, 6 \mathrm{~b}_{1}^{0}$, and $1_{1}^{0}$ vibronic bands which show the spacing of 454,480 , and $656 \mathrm{~cm}^{-1}$ from the origin band, respectively, because of the excellent agreement with those of an $a b$ initio calculation. The well known in-plane C-C-C ring deformation vibrational modes $\mathbf{6 a}$ and $\mathbf{6 b}$ are degenerate in benzene and 1,3,5-trimethylbenzene. ${ }^{29}$ With substitution on the benzene ring, these modes are split, providing lower and higher vibrational frequencies for modes $\mathbf{6 a}$ and $\mathbf{6 b}$, respectively, for Cs symmetry species, while the trend is reversed for $C_{2 \mathrm{v}}$ symmetry. The splitting between these two modes increases with increasing mass of the substituents. The vibrational mode 1 of ring breathing is also the most eminent evidence for identification of benzyl-type radicals and is less sensitive to the substitution, which can be verified in 2,6-dimethylbenzyl radicals.

The band with moderate intensity at $20844 \mathrm{~cm}^{-1}$ was assigned to the origin band of the 2,3,4-trimethylbenzyl radical due to the presence of a series of vibronic and torsional structures in the vicinity of the origin band. Bands belonging to modes $6 \mathbf{a}$ and $\mathbf{6 b}$ were observed at 20384 and $20356 \mathrm{~cm}^{-1}$, with the spacing of 460 and $488 \mathrm{~cm}^{-1}$ from the origin band, respectively. The vibrational mode frequencies of these bands showed excellent agreement with those obtained from the calculation, as listed in Table 2. Thus, the solid state observation of fluorescence bands at 489.9 and $515.5 \mathrm{~nm}$ should be reassigned to the origin band of the 2,3,4- and 2,3,6-trimethylbenzyl radicals, respectively, because of the excellent agreement with those obtained in this work. Both bands showed almost the same deviation from those of the gas phase observation, as described above. Table 3 shows the list of the observed vibronic bands and their assignments.

We also observed the bands belonging to 2,6-dimethylbenzyl radicals which could be generated from 2,3,6trimethylbenzyl radical by removing the methyl group at the meta-position. The mechanism of internal rearrangement in the removal of a substituent has been described to explain the formation of other types of benzyl radicals. The observation of $o$-chlorobenzyl radical in the corona discharge of 2,6-dichlorotoluene is the strong evidence of internal rearrangement of benzyl-type radicals at the excited electronic state. ${ }^{30}$ The recent spectroscopic observation of 3,5-difluorobenzyl radical suggests the involvement of a few pathways in the production of more stable isomers through internal rearrangement of fluorine and methylene groups because the 3,5-difluorobenzyl radical shows the least stability among isomers. ${ }^{31}$ We also observed weak bands in the vicinity of the strong vibronic bands, in which the distance from the strong band is always $42 \mathrm{~cm}^{-1}$, which strongly suggests that these belong to torsional transition during jet expansion, i.e., the torsional motion of the methyl group. $^{10}$

In summary, the vibronic emission spectrum of the benzyl-

Table 2. Vibrational Mode Frequencies $\left(\mathrm{cm}^{-1}\right)$ of Trimethylbenzyl Radicals ${ }^{a}$

\begin{tabular}{|c|c|c|c|c|c|}
\hline Mode $^{b}$ & $\begin{array}{l}\text { This work } \\
\left(\mathrm{D}_{0}\right)\end{array}$ & $\begin{array}{l}\text { Previous work } \\
\qquad\left(\mathrm{D}_{0}\right)\end{array}$ & $\begin{array}{c}\text { Ab initio }{ }^{d} \\
\text { DFT/6-311G** }\left(\mathrm{D}_{0}\right)\end{array}$ & $\begin{array}{c}\text { Precursor } \\
\text { 1,2,3,4-tetramethylbenzene }{ }^{e}\left(\mathrm{~S}_{0}\right)\end{array}$ & $\begin{array}{l}\text { Symmetry } \\
\left(\mathrm{C}_{\mathrm{S}}\right)\end{array}$ \\
\hline \multicolumn{6}{|c|}{ 2,3,4-trimethylbenzyl } \\
\hline origin & 20844 & 20480 & & & \\
\hline $6 a$ & 460 & & 470 & & $a^{\prime}$ \\
\hline $6 \mathbf{b}$ & 488 & & 489 & 445 & $a^{\prime}$ \\
\hline 1 & & & 638 & 752 & $a^{\prime}$ \\
\hline \multicolumn{6}{|c|}{ 2,3,6-trimethylbenzyl } \\
\hline origin & 19796 & 18948 & & & \\
\hline $6 \mathbf{a}$ & 454 & & 462 & & $a^{\prime}$ \\
\hline $\mathbf{6 b}$ & 480 & & 488 & 445 & $a^{\prime}$ \\
\hline 1 & 656 & & 664 & 752 & $a^{\prime}$ \\
\hline
\end{tabular}

${ }^{a}$ Measured in vacuum $\left(\mathrm{cm}^{-1}\right) .{ }^{b}$ Reference $32 .{ }^{c}$ In the solid state, reference $1 .{ }^{d}$ Not scaled. ${ }^{e}$ Reference 29. 
Table 3. List of the Vibronic Bands Observed and Their Assignments

\begin{tabular}{|c|c|c|c|}
\hline Position $^{a}$ & Intensity & $\begin{array}{l}\text { Spacing from the } \\
\text { origin }^{b}\end{array}$ & Assignments $^{c}$ \\
\hline 21594 & $\mathrm{w}$ & & \\
\hline 20844 & $\mathrm{~m}$ & $0(1)$ & origin of $2,3,4-(1)$ \\
\hline 20802 & $\mathrm{w}$ & $42(1)$ & (1) \\
\hline 20618 & $\mathrm{~m}$ & & 2,6-dimethyl \\
\hline 20574 & $\mathrm{w}$ & & $\mathrm{H}$ atomic \\
\hline 20384 & $\mathrm{w}$ & $460(1)$ & $6 a_{1}^{0}(1)$ \\
\hline 20364 & $\mathrm{w}$ & & \\
\hline 20356 & $\mathrm{w}$ & $488(1)$ & $6 b_{1}^{0}(1)$ \\
\hline 20142 & $\mathrm{~m}$ & & 2,6-dimethyl \\
\hline 19940 & $\mathrm{~m}$ & & He atomic \\
\hline 19796 & vs & $0(2)$ & origin of $2,3,6-(2)$ \\
\hline 19754 & $\mathrm{~m}$ & $42(2)$ & $\alpha(2)$ \\
\hline 19342 & $\mathrm{~s}$ & $454(2)$ & $6 a_{1}^{0}(2)$ \\
\hline 19316 & $\mathrm{~s}$ & $480(2)$ & $6 b_{1}^{0}(2)$ \\
\hline 19280 & $\mathrm{w}$ & $516(2)$ & $6 b_{1}^{0}+\alpha(2)$ \\
\hline 19140 & $\mathrm{~s}$ & $656(2)$ & $1_{1}^{0}(2)$ \\
\hline 19100 & $\mathrm{w}$ & $696(2)$ & $1_{1}^{0}+\alpha(2)$ \\
\hline 19068 & $\mathrm{w}$ & $728(2)$ & $6 a_{2}^{0} 1_{1}^{0}(2)$ \\
\hline 18886 & w & $910(2)$ & $6 a_{2}^{0}(2)$ \\
\hline 18862 & w & $934(2)$ & $6 a_{1}^{0} 6 b_{1}^{0}(2)$ \\
\hline 18834 & $\mathrm{w}$ & $962(2)$ & $6 b_{2}^{0}(2)$ \\
\hline 18686 & $\mathrm{w}$ & $1110(2)$ & $6 a_{1}^{0} 1_{1}^{0}(2)$ \\
\hline 18660 & $\mathrm{w}$ & $1136(2)$ & $6 b_{1}^{0} 1_{1}^{0}(2)$ \\
\hline 18580 & $\mathrm{w}$ & $1216(2)$ & $3_{1}^{0}(2)$ \\
\hline 18524 & $\mathrm{~m}$ & $1272(2)$ & \\
\hline 18484 & $\mathrm{w}$ & 1312(2) & $1_{2}^{0}(2)$ \\
\hline 18232 & $\mathrm{w}$ & $1564(2)$ & $6 a_{2}^{0} 1_{1}^{0}(2)$ \\
\hline
\end{tabular}

${ }^{a}$ Measured in vacuum $\left(\mathrm{cm}^{-1}\right) .{ }^{b}$ The numbers 1 and 2 in parentheses indicate the spacing from the origin band in the $D_{1} \rightarrow D_{0}$ transitions of the 2,3,4- and 2,3,6-trimethylbenzyl radicals, respectively. ${ }^{c}$ The numbers 1 and 2 in parentheses indicate the bands belonging to the $D_{1} \rightarrow D_{0}$ transitions of the 2,3,4- and 2,3,6-trimethylbenzyl radicals, respectively. The Greek letters indicate the torsional bands belonging to the strong bands.

type radicals in the $\mathrm{D}_{1} \rightarrow \mathrm{D}_{0}$ transition were recorded in the visible region with a CESE system from the corona discharge of precursor 1,2,3,4-tetramethylbenzene seeded in a large amount of inert carrier gas helium using a pinhole-type glass nozzle. Through the comparison with the data obtained from ab initio calculations using the Gaussian program, we identified the evidence of two isomeric trimethylbenzyl radicals generated in the corona discharge of the precursor. The origin bands and other vibronic bands belonging to the two isomers were clearly identified by comparison with those from $a b$ initio calculations, as well as those from the known data of the precursor.

Acknowledgments. This work was supported by the National Research Foundation of Korea (NRF) grant funded by the Korea Government (MEST) (No. 2008-0061508).

\section{References and Notes}

1. Branciard-Larcher, C.; Migirdicyan, E. Chem. Phys. 1973, 2, 95.

2. Schuler, H.; Reinbeck, L.; Kaberle, A. R. Z. Naturforsh 1952, 7A, 421.

3. Walker, S.; Barrow, R. F. Trans. Faraday Soc. 1954, 50, 541.

4. Bindley, T. F.; Watts, A. T.; Watts, S. Trans. Faraday Soc. 1962, 58,849 .

5. Bindley, T. F.; Watts, A. T.; Watts, S. Trans. Faraday Soc. 1964, $60,1$.

6. Carlton, T. R.; Thrush, B. A. Chem. Phys. Lett. 1986, 125, 547.

7. Hiratsuka, H.; Mori, K.; Shizuke, H.; Fukushima, M.; Obi, K. Chem. Phys. Lett. 1989, 157, 35.

8. Cossart-Magos, C.; Leach, S. J. Chem. Phys. 1976, 64, 4006.

9. Fukushima, M.; Obi, K. J. Chem. Phys. 1990, 93, 8488.

10. Suh, M. H.; Lee, S. K.; Miller, T. A. J. Mol. Spectrosc. 1999, 194, 211.

11. Lin, T.-Y. D.; Miller, T. A. J. Phys. Chem. 1990, 94, 3554.

12. Selco, J. I.; Carrick, P. G. J. Mol. Spectrosc. 1989, 137, 13.

13. Selco, J. I.; Carrick, P. G. J. Mol. Spectrosc. 1995, 173, 277.

14. Lee, G. W.; Lee, S. K. J. Chem. Phys. 2007, 126, 214308.

15. Lee, G. W.; Lee, S. K. J. Phys. Chem. A 2007, 111, 6003.

16. Ahn, H. G.; Lee, G. W.; Kim, T. K.; Lee, S. K. Chem. Phys. Lett. 2008, 465, 193.

17. Petruska, J. J. Chem. Phys. 1961, 34, 1111.

18. Engelking, P. C. Rev. Sci. Instrum. 1986, 57, 2274.

19. Droege, A. T.; Engelking, P. C. Chem. Phys. Lett. 1983, 96, 316.

20. Lee, S. K. Chem. Phys. Lett. 2002, 358, 110.

21. Han, M. S.; Choi, I. S.; Lee, S. K. Bull. Korean Chem. Soc. 1996, $17,991$.

22. Weise, M. L.; Smith, M. W.; Glennon, B. M. Atomic Transition Probabilities; NSRD-NBS4; NBS: Gaithsburg, MD, 1966.

23. Cossart-Magos, C.; Cossart, D. Mol. Phys. 1988, 65, 627.

24. Banwell, C. N.; McCash, E. M. Fundamentals of Molecular Spectroscopy, $4^{\text {th }}$ ed.; McGraw-Hill: New York, NY, 1994.

25. Fujiwara, M.; Tanimoto, Y. J. Phys. Chem. 1994, 98, 5695.

26. Lejeune, V.; Despres, A.; Fourmann, B.; Benoist d'Azy, O.; Migirdicyan, E. J. Phys. Chem. 1987, 91, 6620.

27. Lee, S. K.; Baek, D. Y. Chem. Phys. Lett. 1999, 304, 39.

28. Ahn, H. G.; Lee, G. W.; Kim, T. K.; Lee, S. K. Bull. Korean Chem. Soc. 2008, 29, 2341.

29. Varsanyi, G. Assignments for Vibrational Spectra of Seven Hundred Benzene Derivatives; John Wiley \& Sons: New York, NY, 1974.

30. Yoon, Y. W.; Lee, S. W.; Lee, S. K. Bull. Korean Chem. Soc. 2010, 31, 2479.

31. Yoon, Y. W.; Lee, S. W.; Lee, S. K. Bull. Korean Chem. Soc. 2010, $31,2783$.

32. Wilson, E. B. Phys. Rev. 1934, 45, 706. 